\title{
Dosimetric impact of multileaf collimator leaf width according to sophisticated grade of technique in the IMRT and VMAT planning for pituitary adenoma lesion
}

\author{
Soo-Min Chae ${ }^{1}$, Ki Woong Lee ${ }^{1}$, Seok Hyun Son ${ }^{2}$ \\ ${ }^{1}$ Department of Radiation Oncology, Cheju Halla General Hospital, Jeju, Korea \\ ${ }^{2}$ Department of Radiation Oncology, Incheon St. Mary's Hospital, College of Medicine, The Catholic University of Korea, \\ Seoul, Korea \\ Correspondence to: Seok Hyun Son, email: sonshyun@catholic.ac.kr \\ Keywords: multi-leaf collimator, radiosurgery, intensity-modulated radiotherapy, volumetric modulated arc therapy \\ Received: August 23, 2016 \\ Accepted: October 14, 2016 \\ Published: October 28, 2016
}

\section{ABSTRACT}

We analyzed the difference in the dosimetric effect between 5- $\mathrm{mm}$ and $2.5-\mathrm{mm}$ multileaf collimator (MLC) leaf width according to the sophisticated grades of intensitymodulated radiotherapy (IMRT) and volumetric-modulated arc therapy (VMAT). Nineteen patients with pituitary adenomas were selected for this study. The treatment plans were performed according to the size of the MLC (5-mm and 2.5-mm MLC), the type of technique (IMRT and VMAT), and the sophisticated grades of each technique (5-field, 9-field, 13-field, 17-field technique in IMRT and 1-arc and 2-arc techniques in VMAT). The downsizing effects of MLC leaf width were analyzed using target volume coverage (TVC), conformity index (CI), dose gradient index (GI), and normal tissue difference $\mathbf{7 0} \%$ isodose line and $50 \%$ isodose line. Upon replacing the 5-mm MLC with the 2.5-mm MLC, TVC and CI improved by $1.30 \%$ and $1.36 \%$, respectively, in total plans. The TVC and CI improved by $1.68 \%$ and $1.67 \%$ in IMRT, respectively, and by $0.54 \%$ and $0.72 \%$ in VMAT, respectively. TVC improved by $2.53 \%, 1.82 \%, 1.34 \%$, and $0.94 \%$, and CI also improved by $2.70 \%, 1.81 \%, 1.24 \%$, and $0.94 \%$, in 5-field, 9 -field, 13 -field, and 17-field IMRT, respectively. TVC improved by $0.66 \%$ and $0.43 \%$, and CI also improved by $0.93 \%$, and $0.52 \%$ in 1 -arc and 2 -arc VMAT, respectively. Regarding the target coverage, there were dosimetric benefits of a smaller MLC leaf width. However, the downsizing effect of the MLC leaf width decreased with the use of a more precise RT technique and a more sophisticated grade of the same technique.

\section{INTRODUCTION}

Radiotherapy (RT) has been optimized to enable the delivery of a higher dose of radiation to the target volume in order to enhance the treatment results and lower the dose to the surrounding normal tissues to minimize radiationrelated complications. Radiosurgery is a leading technique within RT, and includes procedures such as 3-dimensional conformal radiotherapy (3DCRT), dynamic conformal arc therapy (DCAT), and intensity-modulated radiotherapy (IMRT). With development of these planning techniques, the multi-leaf collimator (MLC), which make a shape of the irradiated field to deliver radiation to the target volume, was also developed. The MLC leaf width can be made smaller to create a precise irradiated field acceptable for use in accurate radiosurgery. This micro-MLC has reported to be suitable for radiosurgery in terms of both target volume coverage and normal tissues sparing, regardless of which of the aforementioned techniques is used [1-7].

Generally, IMRT has been considered to be more precise than 3DCRT and DCAT, and the downsizing effect of MLC leaf width is more prominent in 3D-CRT or DCAT than in IMRT [3-6]. Therefore, the sophisticated planning technique may substitute a portion of the downsizing effect of MLC leaf width. In previously reported studies, the downsizing effect of MLC leaf width were compared with respect to the different types of techniques only. Thus, there has been no comparison according to the various sophisticated grades of technique within a particular type of technique [2-6]. Therefore, changing the sophisticated grade within the same technique is necessary in order to evaluate the change of the downsizing effect of MLC leaf 
width according to the sophisticated grade of technique. In addition, there have been several reports indicating that the downsizing effect of MLC leaf width differs according to the complexity of the target shape $[4,8-10]$. Thus, to reduce this kind of bias, relatively simple shapes should be selected for evaluation with respect to the target volume.

In this study, we aimed to verify the downsizing effect of MLC leaf width in both IMRT and volumetric modulated arc therapy (VMAT) in patients with pituitary adenoma, which has a spherical shape, using a 5-mm and 2.5-mm MLC. The downsizing effect of MLC leaf width was analyzed using 4 sophisticated grades of IMRT and 2 sophisticated grades of VMAT within each technique.

\section{RESULTS}

\section{The downsizing effect of MLC leaf width in overall treatment plans}

When using 2.5-mm MLC instead of 5.0-mm MLC, TVC and CI improved by $1.30 \% \pm 0.88 \%$ and $1.36 \% \pm$ $0.98 \%$, respectively. GI improved by $8.67 \% \pm 6.65 \%$, and $\mathrm{NTD}_{70}$ and $\mathrm{NTD}_{50}$ were $0.92 \pm 1.34 \mathrm{~cm}^{3}$ and $1.96 \pm 2.28$ $\mathrm{cm}^{3}$, respectively (Table 1 ).

\section{The downsizing effect of MLC leaf width between IMRT and VMAT}

When comparing the downsizing effect of MLC leaf width in IMRT and VMAT, TVC improved by 1.68 $\pm 0.84 \%$ with IMRT and $0.54 \pm 0.26 \%$ with VMAT $(p$ $<0.001)$, and CI improved by $1.67 \pm 1.02 \%$ with IMRT and $0.72 \pm 0.45 \%$ with VMAT $(\mathrm{p}<0.001)$. Regarding target coverage, the improvement ratio was smaller in VMAT than in IMRT, and the difference was statistically significant.

However, GI improved by $9.10 \pm 7.30 \%$ with IMRT and $7.82 \pm 5.11 \%$ with VMAT $(\mathrm{p}=0.520)$. $\mathrm{NTD}_{70}$ was $0.99 \pm 1.45 \mathrm{~cm}^{3}$ with IMRT and $0.78 \pm 0.96 \mathrm{~cm}^{3}$ with VMAT $(p=0.552)$, and $\mathrm{NTD}_{50}$ was $2.11 \pm 2.47 \mathrm{~cm}^{3}$ with IMRT and $1.66 \pm 1.81 \mathrm{~cm}^{3}$ with $\operatorname{VMAT}(\mathrm{p}=0.211)$. Regarding normal tissue sparing, there was no statistically significant difference of improvement ratio between IMRT and VMAT (Table 1 and Figure 1).

\section{The downsizing effect of MLC leaf width according to sophisticated grade of same technique}

\section{IMRT}

By dividing IMRT plans into 5-field, 9-field, 13-field, and 17-field, according to sophisticated grade of treatment technique, TVC improved by $2.53 \pm 0.74 \%, 1.82$ $\pm 0.68 \%, 1.34 \pm 0.57 \%$, and $1.02 \pm 0.50 \%$ with 5 -field, 9-field, 13-field, and 17-field, respectively ( $\mathrm{p}<0.001)$. CI improved by $2.70 \pm 1.10 \%, 1.81 \pm 0.61,1.24 \pm 0.53 \%$, and $0.94 \pm 0.48 \%$ with 5-field, 9-field, 13-field, and 17-field, respectively $(\mathrm{p}<0.001)$. With respect to target coverage, there was a statistically significant grade-dependent decrease with the use of a more sophisticated grade of technique from 5-field to 17-field.

With respect to normal tissue sparing, GI improved by $9.97 \pm 8.10 \%, 9.54 \pm 9.42 \%, 9.00 \pm 5.54 \%$, and 7.88 $\pm 5.85 \%$, with 5-field, 9-field, 13-field, and 17-field, respectively $(p=0.363)$, and there was no statistically significant difference. $\mathrm{NTD}_{70}$ were $0.77 \pm 1.47 \mathrm{~cm}^{3}$, $1.26 \pm 2.15 \mathrm{~cm}^{3}, 0.93 \pm 1.02 \mathrm{~cm}^{3}$, and $1.02 \pm 1.18 \mathrm{~cm}^{3}$ with 5-field, 9-field, 13-field, and 17-field, respectively $(\mathrm{p}=0.166)$ and $\mathrm{NTD}_{50}$ were $1.56 \pm 2.32 \mathrm{~cm}^{3}, 2.03 \pm 2.15$ $\mathrm{cm}^{3}, 2.35 \pm 2.65 \mathrm{~cm}^{3}$, and $2.51 \pm 2.91 \mathrm{~cm}^{3}$ with 5-field, 9-field, 13-field, and 17-field, respectively ( $p=0.671)$. There was no statistically significant difference according to the sophisticated grades of this technique (Table 1 and Figure 1).

\section{VMAT}

By dividing VMAT plans into 1-arc and 2-arc, according to sophisticated grade of treatment technique, TVC improved by $0.66 \pm 0.27 \%$ and $0.43 \pm 0.20 \%$ with 1 -arc and 2-arc, respectively $(\mathrm{p}<0.001)$, and CI improved by $0.93 \pm 0.46 \%$ and $0.52 \pm 0.33 \%$ with 1 -arc and 2 -arc, respectively $(\mathrm{p}<0.001)$. With respect to target coverage, there was a statistically significant decrease with the use of a more sophisticated grade of technique from 1-arc to 2-arc.

With respect to normal tissue sparing, GI improved by $7.93 \pm 5.46 \%$ and $7.72 \pm 4.87 \%$ with 1 -arc and 2 -arc, respectively $(\mathrm{p}=0.936)$. $\mathrm{NTD}_{70}$ was $0.67 \pm 1.07 \mathrm{~cm}^{3}$ and $0.90 \pm 0.85 \mathrm{~cm}^{3}$ with 1 -arc and 2-arc, respectively $(\mathrm{p}=0.530)$, and $\mathrm{NTD}_{50}$ was $1.41 \pm 1.85 \mathrm{~cm}^{3}$ vs. $1.91 \pm$ $1.78 \mathrm{~cm}^{3}$ with 1 -arc and 2-arc, respectively $(\mathrm{p}=0.990)$. There was no statistically significant difference according to sophisticated grade of treatment technique (Table 1 and Figure 1).

\section{DISCUSSION}

Radiosurgery is a treatment technique used for delivering highly eradicating radiation dose to the target volume and sparing the surrounding normal tissues [2, 11-13]. Several studies have reported that pa prescribed dose of 20-29 Gy to a pituitary adenoma resulted in an 82$100 \%$ tumor control rate, and therefore, in this planning study, we used a prescribed dose of 25 Gy to the target volume [14-18].

MLC, which have undergone continual development in terms of field size and tungsten leaf width over the previous 2 decades, have been classified into standard-MLC with a width greater than $5 \mathrm{~mm}$, and 
Table 1: Dosimetric improvement ratio according to planning technique and sophisticated grade of each technique

\begin{tabular}{|c|c|c|c|c|c|c|c|c|c|c|c|c|}
\hline \multirow[b]{2}{*}{ Index } & \multirow[b]{2}{*}{ Total } & \multicolumn{3}{|c|}{ IMRT vs. VMAT } & \multicolumn{5}{|c|}{ IMRT } & \multicolumn{3}{|c|}{ VMAT } \\
\hline & & IMRT & VMAT & $\underset{\text { value }^{1}}{\mathbf{p}}$ & 5-field & 9-field & 13-field & 17-field & $\begin{array}{c}\text { p } \\
\text { value }^{2}\end{array}$ & 1 -arc & 2 -arc & $\underset{\text { value }^{3}}{\mathbf{p}}$ \\
\hline TVC IR (\%) & $1.30 \pm 0.88$ & $1.68 \pm 0.84$ & $0.54 \pm 0.26$ & $<0.001$ & $2.53 \pm 0.74$ & $1.82 \pm 0.68$ & $1.34 \pm 0.57$ & $1.02 \pm 0.50$ & $<0.001$ & $0.66 \pm 0.27$ & $0.43 \pm 0.20$ & $<0.001$ \\
\hline CI IR (\%) & $1.36 \pm 0.98$ & $1.67 \pm 1.02$ & $0.72 \pm 0.45$ & $<0.001$ & $2.70 \pm 1.10$ & $1.81 \pm 0.61$ & $1.24 \pm 0.53$ & $0.94 \pm 0.48$ & $<0.001$ & $0.93 \pm 0.46$ & $0.52 \pm 0.33$ & $<0.001$ \\
\hline GI IR (\%) & $8.67 \pm 6.65$ & $9.10 \pm 7.30$ & $7.82 \pm 5.11$ & 0.520 & $9.97 \pm 8.10$ & $9.54 \pm 9.42$ & $9.00 \pm 5.54$ & $7.88 \pm 5.85$ & 0.363 & $7.93 \pm 5.46$ & $7.72 \pm 4.87$ & 0.936 \\
\hline $\mathrm{NTD}_{70}\left(\mathrm{~cm}^{3}\right)$ & $0.92 \pm 1.34$ & $0.99 \pm 1.45$ & $0.78 \pm 0.96$ & 0.552 & $0.77 \pm 1.47$ & $1.26 \pm 2.15$ & $0.93 \pm 1.02$ & $1.02 \pm 1.18$ & 0.166 & $0.67 \pm 1.07$ & $0.90 \pm 0.85$ & 0.530 \\
\hline $\mathrm{NTD}_{50}\left(\mathrm{~cm}^{3}\right)$ & $1.96 \pm 2.28$ & $2.11 \pm 2.47$ & $1.66 \pm 1.81$ & 0.211 & $1.56 \pm 2.32$ & $2.03 \pm 2.15$ & $2.35 \pm 2.65$ & $2.51 \pm 2.91$ & 0.671 & $1.41 \pm 1.85$ & $1.91 \pm 1.78$ & 0.990 \\
\hline
\end{tabular}

Abbreviations: IMRT = intensity-modulated radiotherapy; VMAT $=$ volumetric-modulated arc therapy; $\mathrm{TVC}=$ target volume coverage; $\mathrm{CI}=$ conformity index; GI = dose gradient index; IR = improvement ratio; $\mathrm{NTD}_{70}=$ normal tissue difference of $70 \%$ isodose line; $\mathrm{NTD}_{50}=$ normal tissue difference of $50 \%$ isodose line.

${ }^{1}$ independent t-test.

${ }^{2}$ paired Friedman test.

${ }^{3}$ Wilcoxin signed rank test.

mini-MLC, which has a width below $4 \mathrm{~mm}$. StandardMLC (5-mm) has been commonly used in conventional RT, whilst 2.5-mm micro-MLC has been used for radiosurgery. As a smaller MLC is able to yield a precise shape for the irradiated field, several studies have reported that downsizing MLC leaf width displayed dosimetric superiority and clinical effectiveness [1-7, 9, 19-22]. Since the development of 4-mm MLC leaf width, several studies have compared the use of $10-\mathrm{mm}$ MLC with that of 4-mm MLC in IMRT [19-22]. Gong et al. reported that, in terms of target coverage, CI and HI improved by $1.1-4.3 \%$ and $1.1-1.5 \%$, respectively, and regarding normal tissue sparing, $\mathrm{D}_{5}$ of spinal cord, $\mathrm{V}_{10}$ and $\mathrm{V}_{20}$ of lung, and mean lung dose improved by $4.2 \%, 2.4 \%, 3.6 \%$ and $2.4 \%$, respectively [21]. Wang et al. reported that, in terms of target coverage, CI and HI improved by $0.9-4.8 \%$ and $0.7-0.8 \%$, respectively, whilst with regard to normal tissue sparing, there were no statistically significant difference [22]. Since the size of standard-MLC leaf width was changed from $10 \mathrm{~mm}$ to $5 \mathrm{~mm}$, and 2.5-mm micro-MLC has been commonly used in radiosurgery, several studies have reported the effectiveness of 2.5-mm MLC compared with 5-mm MLC $[3-5,7]$. Tanyi et al. reported the impact of MLC on 3DCRT, DCAT, and IMRT in 11 cases of liver and 18 cases of lung tumors. With regard to target coverage, CI improved in $62.1 \%, 55.2 \%$ and $51.7 \%$ of patients treated with the 3DCRT, DCAT, and IMRT with the use of a 2.5-mm MLC. With respect to normal tissue sparing, volume of normal tissue receiving more than $90 \%$ / $50 \%$ of the prescription dose was decreased in $72.4 \%$ / 86.2\%, 69.0\% / 79.3\%, and 65.5\% / 75.9\% patients treated with 3D-CRT, DCAT, and IMRT, respectively, in 2.5-mm MLC group [5]. A further study by Tanyi et al. regarding 68 brain radiosurgeries showed that with respect to target coverage, CI improved by $5 \%, 2.1 \%$, and $1.5 \%$ with DCAT, 3DCRT, and IMRT, respectively.
With respect to normal tissue sparing, GI improved by $11.7 \%, 6.4 \%$, and $4.8 \%$ with DCAT, 3DCRT, and IMRT, respectively, and peritumoral rind volume receiving $50 \%$ of the prescribed dose (PRV50) improved by $18.8 \%, 12.1 \%$, and $7.2 \%, \%$ with DCAT, 3DCRT, and IMRT, respectively, in the group who received treatment with 2.5-mm MLC [3]. In this study, TVC and CI improved by $1.30 \%$ and $1.36 \%$, respectively when the 2.5-mm MLC was used instead of the 5.0-mm MLC. These results are consistent with the studies previously mentioned above.

The downsizing effect of MLC leaf varied dependent on the treatment technique applied, such as 3DCRT, DCAT, and IMRT, and the effect was most decreased in IMRT, which is more sophisticated technique than 3DCRT and DCAT $[3,5]$. In the case of VMAT, single-arc VMAT was similar to multiple staticIMRT; however, multiple-arc VMAT was superior to multiple static-IMRT [23-28]. The downsizing effect of MLC leaf width has predominantly been reported for 3DCRT, DCAT, and IMRT. There have only been a few reports concerning the downsizing effect of MLC leaf width in VMAT $[10,29,30]$. Our previous study regarding the downsizing effect of MLC leaf width in spinal radiosurgery revealed that TVC improved by $8.38 \%$ and $2.97 \%$ in IMRT and VMAT, respectively, and the effect was smaller with VMAT than with IMRT [10]. In this study comparing IMRT with VMAT, TVC improved by $1.68 \%$ and $0.54 \%$ with IMRT and VMAT, respectively, and $\mathrm{CI}$ improved by $1.67 \%$ and $0.72 \%$ with IMRT and VMAT, respectively. The downsizing effect of MLC leaf width was found to be smaller in VMAT than IMRT.

In addition, in this study, the downsizing effect of MLC leaf width according to the sophisticated grade of each technique was evaluated. When 2.5-mm MLC was used instead of 5.0-mm MLC, TVC improved by $2.53 \%$, 
$1.82 \%, 1.34 \%$, and $0.94 \%$ in 5-field, 9-field, 13-field, and 17-field IMRT, respectively, and CI also improved by $2.70 \%, 1.81 \%, 1.24 \%$, and $0.94 \%$, in 5-field, 9 -field, 13-field, and 17-field IMRT, respectively. These results revealed that the downsizing effect of MLC leaf width decreased with the use of a more sophisticated grade of technique from 5-field to 17-field technique. In the case of VMAT, TVC improved by $0.66 \%$ and $0.43 \%$ in 1-arc and 2-arc VMAT, respectively, and CI also improved by $0.93 \%$, and $0.52 \%$ in 1 -arc and 2 -arc VMAT, respectively, which revealed that the downsizing effect of MLC leaf width decreased with the use of a more sophisticated grade of technique from 1-arc to 2-arc VMAT. With respect to normal tissue sparing, there were no statistically significant difference according to the sophisticated grade of technique used (IMRT or VMAT). This was because the present study was designed to obtain the maximal target coverage, limiting the dose delivered to $10 \mathrm{~Gy}$ for the optic chiasm, and $15 \mathrm{~Gy}$ for the brainstem in all plans.

In conclusion, with respect to the target coverage, there were dosimetric benefits of a smaller MLC leaf width, which concurs with previously reported studies. However, the downsizing effect of the MLC leaf width decreased with the use of a more precise RT technique and a more sophisticated grade of the same technique. With the uses of IMRT or VMAT, this effect was very small, with less than 3\% improvement ratio, and less than $1 \%$ with VMAT. Therefore, further studies are required regarding the necessity for the use of smaller MLC leaf width in the real treatment.

\section{MATERIALS AND METHODS}

\section{Patient selection}

The present study was designed to evaluate the downsizing effect of MLC leaf width according to sophisticated grade of RT technique in IMRT and VMAT by comparing the dose distribution with the use of 5-mm MLC and 2.5-mm MLC. Nineteen patients with pituitary adenomas were selected for this study due to the relatively round shape of the target. Computed tomography (CT) data of all patients were retrospectively collected for this study following institutional review board approval (IRB of Incheon St. Mary's Hospital, the Catholic University of Korea, Reference number: OC16RISI0117).

\section{Simulation and target delineation}

Patients were immobilized using a thermoplastic head mask for the brain prior to simulation. CT scans were performed using the Ingenuity 128-channel CT scanner (Philips Healthcare, Eindhoven, Netherlands) with a 1-mm slice thickness, and Magnetic resonance imaging (MRI) scans were performed with a 1-mm slice thickness. $\mathrm{CT}$ and MRI images were imported onto Eclipse version 8.9 (Varian Medical System, Palo, Alto, CA), and image fusion was performed with registration by using Rigid Registration Algorithm version 8.9.17 using pixel data. Target volume and organs at risk (OARs) such as the optic pathway, optic chiasm, and brain stem were contoured based on MRI images. The mean target volume was 6.27 $\pm 8.83 \mathrm{~cm}^{3}$ (range: $0.158 \mathrm{~cm}^{3}-31.144 \mathrm{~cm}^{3}$ ).
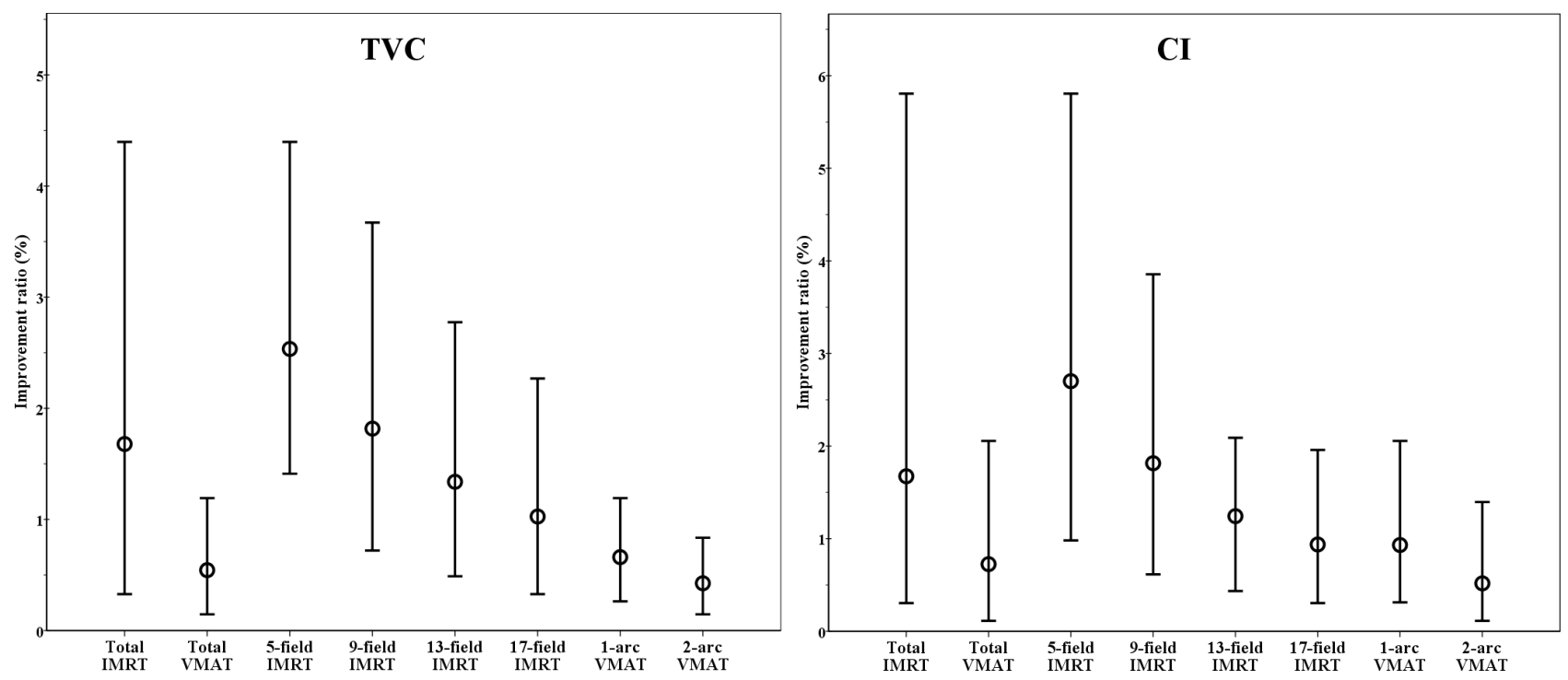

Figure 1: Improvement ratio according to sophisticated grade of each technique for TVC and CI. The points presented in this graph indicate the maximum, average, and minimum improvement ratios.

Abbreviations: $\mathrm{TVC}=$ target volume coverage; $\mathrm{CI}=$ conformity index; IMRT $=$ intensity-modulated radiotherapy; VMAT $=$ volumetricmodulated arc therapy. 


\section{Prescription and radiotherapy planning}

The prescribed dose was 25 Gy in a single fraction, which was required to treat the functional pituitary adenomas, and a dose of $10 \mathrm{~Gy}$ and $8 \mathrm{~Gy}$ was allowed to $0.5 \mathrm{cc}$ of the brain stem and $0.2 \mathrm{cc}$ of the optic chiasm/ pathway, respectively. In this study, we intended to obtain the maximal target volume coverage whilst satisfying the dose constraints for the brain stem and optic chiasm/pathway. Therefore, the irradiated dose to $0.5 \mathrm{cc}$ of the brain stem and $0.2 \mathrm{cc}$ of the optic chiasm/ pathway was almost the same for all treatment plans. However, the coverage rates of the prescribed dose to the target volume varied according to each type of plan. Treatment planning was performed using both $5-\mathrm{mm}$ MLC and 2.5-mm MLC to evaluate the downsizing effect of MLC leaf width. All plans were generated using Eclipse version 8.9 for excluding the bias from different algorithms. For the plan optimization, Dose Volume Optimizer version 8.9 was used for IMRT, and Progressive Resolution Optimizer version 8.9 was used for VMAT. Anisotropic Analytic Algorithm version 8.9 was used for dose calculation. The Fluence map pixel size was $1.5 \times 1.5 \mathrm{~mm}^{2}$.

IMRT plans consisted of single isocenter, coplanar, and static fields delivered by the sliding-window method (dynamic MLC mode), and the whole gantry angles were $0^{\circ}, 72^{\circ}, 144^{\circ}, 216^{\circ}, 218^{\circ}$ in 5-field, $0^{\circ}, 40^{\circ}, 80^{\circ}, 120^{\circ}$, $160^{\circ}, 200^{\circ}, 240^{\circ}, 280^{\circ}, 320^{\circ}$ in 9 -field, $0^{\circ}, 27^{\circ}, 54^{\circ}, 81^{\circ}$, $108^{\circ}, 135^{\circ}, 163^{\circ}, 191^{\circ}, 219^{\circ}, 248^{\circ}, 275^{\circ}, 302^{\circ}, 330^{\circ}$ in 13 -field, and $0^{\circ}, 21^{\circ}, 42^{\circ}, 63^{\circ}, 84^{\circ}, 105^{\circ}, 126^{\circ}, 147^{\circ}$, $168^{\circ}, 189^{\circ}, 210^{\circ}, 231^{\circ}, 252^{\circ}, 273^{\circ}, 294^{\circ}, 316^{\circ}, 338^{\circ}$ in 17 field, respectively.

VMAT plans were implemented with a single isocenter, and whole gantry angles were in the counterclockwise direction 179.9-180.1 ${ }^{\circ}$ in 1-arc, and counterclockwise direction $179.9-180.1^{\circ}$ plus clockwise direction $180.1-179.9^{\circ}$ in 2 -arc, respectively.

Finally, 228 treatment plans were generated according to 6 types of sophisticated grades of technique (4 forms of IMRT plans and 2 forms of VMAT plans) and 2 MLC leaf widths for 19 patients. An example of the generated treatment plans is shown in Figure 2.

\section{Dosimetric indices}

To analyze the treatment plan efficiency, we use dosimetric indices, which included Target Volume Coverage (TVC), Conformal Index (CI), Dose Gradient Index (GI), Normal Tissue Difference 70 and 50 (NTD 70 and 50), and improvement ratio (IR).

1. TVC: The index to evaluate the dose coverage to the target volume $[31,32]$. The ideal TVC is $100 \%$, and a greater value of TVC indicates a superior dose coverage of the target volume.
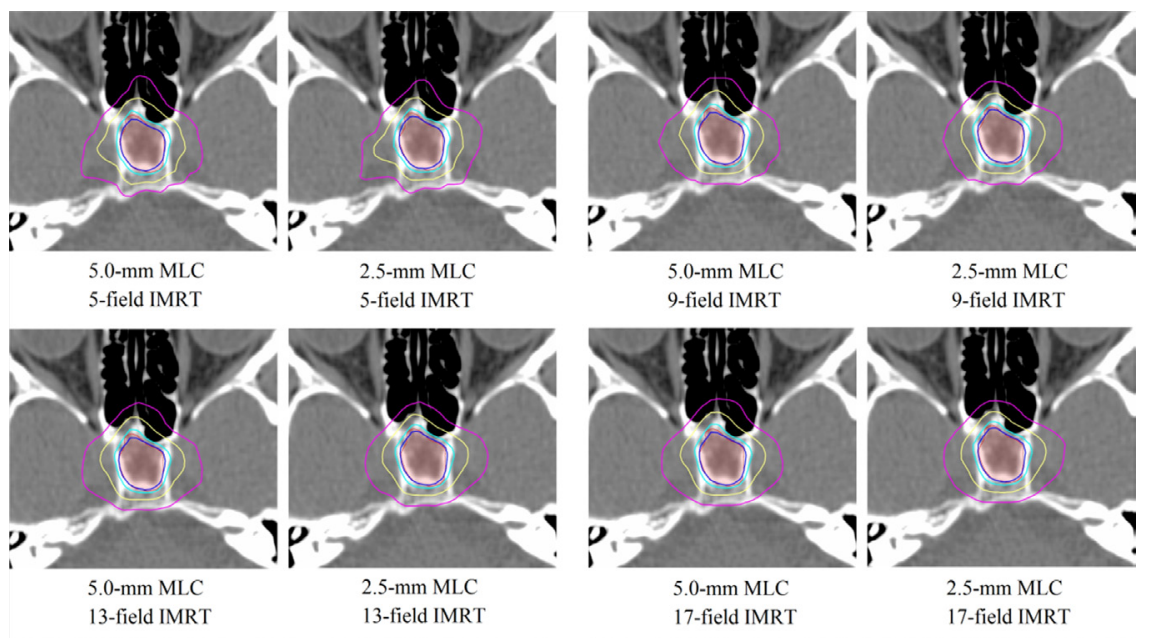

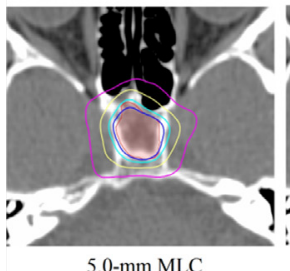

5.0-mm MLC 1-arc VMAT

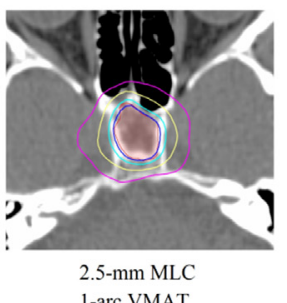

1-arc VMAT

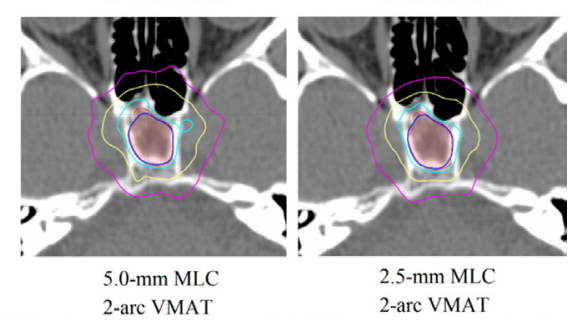

Figure 2: Example of dose distribution in axial plane according to MLC leaf width and sophisticated grades of each technique (the blue line is $\mathbf{1 0 0 \%}$ isodose line, the cyan line is $\mathbf{9 0 \%}$ isodose line, yellow line is $\mathbf{7 0 \%}$ isodose line, purple line is $50 \%$ isodose line).

Abbreviations: MLC = multileaf collimator; IMRT = intensity-modulated radiotherapy; VMAT = volumetric-modulated arc therapy. 


$$
\operatorname{TVC}(\%)=\frac{\begin{array}{l}
\text { Volume within the target receiving at } \\
\text { least the prescription isodose }
\end{array}}{\text { Target volume }} \times 100(\%)
$$

2. CI: The ratio used to evaluate the quality of fit of the target volume to the prescription isodose volume. The ratio was proposed by the Radiation Therapy Oncology Group (RTOG) and modified by Paddick et al. and Nakamura et al. [11,33-37]. The ideal CI is 1 and a smaller value of $\mathrm{CI}$ indicates a better conformity to the target volume.

$$
\text { Conformity index }(\mathrm{CI})=\frac{\mathrm{PIV} \mathrm{TV}^{\prime} \mathrm{TV}}{\mathrm{PTV}_{\mathrm{PIS}} \mathrm{PTV}_{\mathrm{PIS}}}
$$

[PIV, prescription isodose volume; $\mathrm{PTV}_{\mathrm{PIS}}$, planning target volume encompassed within the prescription isodose surface; TV, Target volume]

3. GI: The index that represent the degree of dose drop-off outside the target volume, proposed by Paddick et al. [38]. A smaller GI value indicates a better degree of dose dropoff outside the target volume.

$$
\text { Dose gradient index }(\mathrm{GI})=\frac{\mathrm{V}_{50}}{\mathrm{PTV}_{\mathrm{PIS}}}, 100(\%)
$$

$\left[\mathrm{V}_{50}\right.$, volume receiving at least $50 \%$ of the prescription dose; PTV $_{\text {PIS }}$ planning target volume encompassed within the prescription isodose surface]

4. Normal tissue difference $70 \& 50\left(\mathrm{NTD}_{70} \& \mathrm{NTD}_{50}\right)$ : The index is defined as the difference between the volume of normal tissue receiving a certain dose utilizing $5-\mathrm{mm}$ MLC, and the volume receiving the same dose using 2.5$\mathrm{mm}$ MLC, in order to assess the degree of normal tissue sparing, and was proposed by Dhabaan et al [7]. The positive greater value of $\mathrm{NTD}_{70} \& \mathrm{NTD}_{50}$ indicates a better sparing of normal tissue

$$
\begin{aligned}
& \mathrm{NTD}_{70}=\mathrm{NTD}_{70}{ }^{5-\mathrm{mm} \mathrm{MLC}}-\mathrm{NTD}_{70}{ }^{2.5-\mathrm{mm} \mathrm{MLC}}\left(\mathrm{cm}^{3}\right) \\
& \mathrm{NTD}_{50}=\mathrm{NTD}_{50}{ }^{5-\mathrm{mm} \mathrm{MLC}}-\mathrm{NTD}_{50}{ }^{2.5-\mathrm{mm} \mathrm{MLC}}\left(\mathrm{cm}^{3}\right)
\end{aligned}
$$

$\left[\mathrm{NTV}_{70}\right.$, Volume of normal tissue receiving $70 \%$ of the prescribed isodose; $\mathrm{NTV}_{50}$, Volume of normal tissue receiving $50 \%$ of the prescribed isodose]

5. Improvement ratio: The ratio used to evaluate the improvement in the index between the two plans (a 2.5mm MLC plan vs. 5-mm MLC plan) [39].

$$
\underset{\text { ratio }}{\operatorname{Improvement}}(\%)=\frac{\mid \text { Index }_{2.5}-\mathrm{mm} \mathrm{MLC}^{-} \text {Index }_{5-\mathrm{mm} \mathrm{MLC}} \mid}{\operatorname{Index}_{5-\mathrm{mm} \mathrm{MLC}}} \times 100(\%)
$$

\section{Statistical analysis}

Statistical analysis was conducted using SPSS for Windows, version 18.0 (SPSS Inc., Chicago, IL, USA) and a $p$ value $<0.05$ was considered significant. To analyze the effect of MLC leaf width on entire planning and to compare of IMRT and VMAT planning, independent t-test was used. To evaluate the difference in terms of sophisticated grade of IMRT and VMAT, paired Friedman test and Wilcoxon signed rank test were used, respectively.

\section{CONFLICTS OF INTEREST}

The authors declare that they have no competing interests.

\section{REFERENCES}

1. Monk JE, Perks JR, Doughty D, Plowman PN. Comparison of a micro-multileaf collimator with a 5-mm-leaf-width collimator for intracranial stereotactic radiotherapy. Int $\mathrm{J}$ Radiat Oncol Biol Phys. 2003; 57:1443-1449.

2. Dvorak P, Georg D, Bogner J, Kroupa B, Dieckmann K, Potter R. Impact of IMRT and leaf width on stereotactic body radiotherapy of liver and lung lesions. Int $\mathrm{J}$ Radiat Oncol Biol Phys. 2005; 61:1572-1581.

3. Tanyi JA, Kato CM, Chen Y, Chen Z, Fuss M. Impact of the high-definition multileaf collimator on linear acceleratorbased intracranial stereotactic radiosurgery. Br J Radiol. 2011; 84:629-638.

4. Wu QJ, Wang Z, Kirkpatrick JP, Chang Z, Meyer JJ, Lu M, Huntzinger C, Yin FF. Impact of collimator leaf width and treatment technique on stereotactic radiosurgery and radiotherapy plans for intra- and extracranial lesions. Radiat Oncol. 2009; 4:3.

5. Tanyi JA, Summers PA, McCracken CL, Chen Y, Ku LC, Fuss M. Implications of a high-definition multileaf collimator (HD-MLC) on treatment planning techniques for stereotactic body radiation therapy (SBRT): a planning study. Radiat Oncol. 2009; 4:22.

6. Jin J-Y, Yin F-F, Ryu S, Ajlouni M, Kim JH. Dosimetric study using different leaf-width MLCs for treatment planning of dynamic conformal arcs and intensitymodulated radiosurgery. Medical Physics. 2005; 32:405.

7. Dhabaan A, Elder E, Schreibmann E, Crocker I, Curran WJ, Oyesiku NM, Shu HK, Fox T. Dosimetric performance of the new high-definition multileaf collimator for intracranial stereotactic radiosurgery. J Appl Clin Med Phys. 2010; 11:3040.

8. Burmeister J, McDermott PN, Bossenberger T, Ben-Josef E, Levin K, Forman JD. Effect of MLC leaf width on the planning and delivery of SMLC IMRT using the CORVUS inverse treatment planning system. Medical Physics. 2004; $31: 3187$. 
9. Kubo HD, Wilder RB, Pappas CT. Impact of collimator leaf width on stereotactic radiosurgery and 3D conformal radiotherapy treatment plans. Int J Radiat Oncol Biol Phys. 1999; 44:937-945.

10. Chae SM, Lee GW, Son SH. The effect of multileaf collimator leaf width on the radiosurgery planning for spine lesion treatment in terms of the modulated techniques and target complexity. Radiat Oncol. 2014; 9:72.

11. Nakamura JL, Verhey LJ, Smith V, Petti PL, Lamborn KR, Larson DA, Wara WM, McDermott MW, Sneed PK. Dose conformity of gamma knife radiosurgery and risk factors for complications. Int J Radiat Oncol Biol Phys. 2001; 51:1313-1319.

12. Lindquist C. Gamma Knife Radiosurgery. Semin Radiat Oncol. 1995; 5:197-202.

13. Solberg TD, Boedeker KL, Fogg R, Selch MT, DeSalles AA. Dynamic arc radiosurgery field shaping: a comparison with static field conformal and noncoplanar circular arcs. Int J Radiat Oncol Biol Phys. 2001; 49:1481-1491.

14. Tinnel BA, Henderson MA, Witt TC, Fakiris AJ, Worth RM, Des Rosiers PM, Edmondson JW, Timmerman RD, Lo SS. Endocrine response after gamma knife-based stereotactic radiosurgery for secretory pituitary adenoma. Stereotact Funct Neurosurg. 2008; 86:292-296.

15. Laws ER, Sheehan JP, Sheehan JM, Jagnathan J, Jane JA, Jr., Oskouian R. Stereotactic radiosurgery for pituitary adenomas: a review of the literature. J Neurooncol. 2004; 69:257-272.

16. Sheehan JP, Pouratian N, Steiner L, Laws ER, Vance ML. Gamma Knife surgery for pituitary adenomas: factors related to radiological and endocrine outcomes. J Neurosurg. 2011; 114:303-309.

17. Jagannathan J, Yen CP, Pouratian N, Laws ER, Sheehan JP. Stereotactic radiosurgery for pituitary adenomas: a comprehensive review of indications, techniques and longterm results using the Gamma Knife. J Neurooncol. 2009; 92:345-356.

18. Kajiwara K, Saito K, Yoshikawa K, Ideguchi M, Nomura S, Fujii M, Suzuki M. Stereotactic radiosurgery/radiotherapy for pituitary adenomas: a review of recent literature. Neurol Med Chir (Tokyo). 2010; 50:749-755.

19. Wang L, Movsas B, Jacob R, Fourkal E, Chen L, Price R, Feigenberg S, Konski A, Pollack A, Ma C. Stereotactic IMRT for prostate cancer: dosimetric impact of multileaf collimator leaf width in the treatment of prostate cancer with IMRT. J Appl Clin Med Phys. 2004; 5:29-41.

20. Wang L, Hoban P, Paskalev K, Yang J, Li J, Chen L, Xiong W, Ma CC. Dosimetric advantage and clinical implication of a micro-multileaf collimator in the treatment of prostate with intensity-modulated radiotherapy. Med Dosim. 2005; 30:97-103.

21. Gong Y, Wang S, Zhou L, Liu Y, Xu Y, Lu Y, Bai S, Fu $\mathrm{Y}, \mathrm{Xu}$ Q, Jiang Q. Dosimetric comparison using different multileaf collimeters in intensity-modulated radiotherapy for upper thoracic esophageal cancer. Radiat Oncol. 2010; 5:65.

22. Wang S, Gong Y, Xu Q, Bai S, Lu Y, Jiang Q, Chen N. Impacts of multileaf collimators leaf width on intensitymodulated radiotherapy planning for nasopharyngeal carcinoma: analysis of two commercial elekta devices. Med Dosim. 2011; 36:153-159.

23. Yu CX. Intensity-modulated arc therapy with dynamic multileaf collimation: an alternative to tomotherapy. Phys Med Biol. 1995; 40:1435-1449.

24. Wu QJ, Yoo S, Kirkpatrick JP, Thongphiew D, Yin FF. Volumetric arc intensity-modulated therapy for spine body radiotherapy: comparison with static intensitymodulated treatment. Int J Radiat Oncol Biol Phys. 2009; 75:1596-1604.

25. Yoo S, Wu QJ, Lee WR, Yin FF. Radiotherapy treatment plans with RapidArc for prostate cancer involving seminal vesicles and lymph nodes. Int J Radiat Oncol Biol Phys. 2010; 76:935-942.

26. Tsai CL, Wu JK, Chao HL, Tsai YC, Cheng JC. Treatment and dosimetric advantages between VMAT, IMRT, and helical tomotherapy in prostate cancer. Med Dosim. 2011; 36:264-271.

27. Lee YK, Bedford JL, McNair HA, Hawkins MA. Comparison of deliverable IMRT and VMAT for spine metastases using a simultaneous integrated boost. Br J Radiol. 2013; 86:20120466.

28. Roa DE, Schiffner DC, Zhang J, Dietrich SN, Kuo JV, Wong J, Ramsinghani NS, Al-Ghazi MS. The use of RapidArc volumetric-modulated arc therapy to deliver stereotactic radiosurgery and stereotactic body radiotherapy to intracranial and extracranial targets. Med Dosim. 2012; $37: 257-264$.

29. Serna A, Puchades V, Mata F, Ramos D, Alcaraz M. Influence of multi-leaf collimator leaf width in radiosurgery via volumetric modulated arc therapy and $3 \mathrm{D}$ dynamic conformal arc therapy. Phys Med. 2015; 31:293-296.

30. van Kesteren Z, Janssen TM, Damen E, van VlietVroegindeweij C. The dosimetric impact of leaf interdigitation and leaf width on VMAT treatment planning in Pinnacle: comparing Pareto fronts. Phys Med Biol. 2012; 57:2943-2952.

31. Sankaranarayanan V, Ganesan S, Oommen S, Padmanaban TK, Stumpf J, Ayyangar KM. Study on dosimetric parameters for stereotactic radiosurgery and intensitymodulated radiotherapy. Med Dosim. 2003; 28:85-90.

32. Lomax NJ, Scheib SG. Quantifying the degree of conformity in radiosurgery treatment planning. Int J Radiat Oncol Biol Phys. 2003; 55:1409-1419.

33. Shaw E, Scott C, Souhami L, Dinapoli R, Kline R, Loeffler J, Farnan N. Single dose radiosurgical treatment of recurrent previously irradiated primary brain tumors and 
brain metastases: final report of RTOG protocol 90-05. Int J Radiat Oncol Biol Phys. 2000; 47:291-298.

34. Shaw E, Scott C, Souhami L, Dinapoli R, Bahary JP, Kline R, Wharam M, Schultz C, Davey P, Loeffler J, Del Rowe J, Marks L, Fisher B, Shin K. Radiosurgery for the treatment of previously irradiated recurrent primary brain tumors and brain metastases: initial report of radiation therapy oncology group protocol (90-05). Int J Radiat Oncol Biol Phys. 1996; 34:647-654.

35. Souhami L, Seiferheld W, Brachman D, Podgorsak EB, Werner-Wasik M, Lustig R, Schultz CJ, Sause W, Okunieff P, Buckner J, Zamorano L, Mehta MP, Curran WJ, Jr. Randomized comparison of stereotactic radiosurgery followed by conventional radiotherapy with carmustine to conventional radiotherapy with carmustine for patients with glioblastoma multiforme: report of Radiation Therapy Oncology Group 93-05 protocol. Int J Radiat Oncol Biol Phys. 2004; 60:853-860.
36. Andrews DW, Scott CB, Sperduto PW, Flanders AE, Gaspar LE, Schell MC, Werner-Wasik M, Demas W, Ryu J, Bahary J-P, Souhami L, Rotman M, Mehta MP, Curran WJ. Whole brain radiation therapy with or without stereotactic radiosurgery boost for patients with one to three brain metastases: phase III results of the RTOG 9508 randomised trial. The Lancet. 2004; 363:1665-1672.

37. Paddick I. A simple scoring ratio to index the conformity of radiosurgical treatment plans. Technical note. J Neurosurg. 2000; 93 Suppl 3:219-222.

38. Paddick I, Lippitz B. A simple dose gradient measurement tool to complement the conformity index. J Neurosurg. 2006; 105 Suppl:194-201.

39. Lee TF, Chao PJ, Wang CY, Lan JH, Huang YJ, Hsu HC, Sung CC, Su TJ, Lian SL, Fang FM. Dosimetric comparison of helical tomotherapy and dynamic conformal arc therapy in stereotactic radiosurgery for vestibular schwannomas. Med Dosim. 2011; 36:62-70. 